\title{
A UTILIZAÇÃO DE FERRAMENTAS E TÉCNICAS DE GERÊNCIA DE PROJETOS E GESTÃO DE CONHECIMENTO PARA ESTIMULAR O SUCESSO EM PROJETOS DE INOVAÇÃO
}

USING TOOLS AND TECHNIQUES OF PROJECT MANAGEMENT AND KNOWLEDGE MANAGEMENT TO INCREASE THE SUCCESS OF INNOVATION PROJECTS

LA UTILIZACIÓN DE HERRAMIENTAS Y TÉCNICAS DE GERENCIA EN PROYECTOS Y GESTIÓN DE CONOCIMIENTO PARA ESTIMULAR EL EXITO EN PROYECTOS DE INNOVACIÓN

\author{
ANTONIO RICARDO MENDROT \\ Doutorando \\ Universidade de Taubaté - Brasil \\ ricardo.mendrot@gmail.com
}

EDSON APARECIDA DE ARAUJO QUERIDO OLIVEIRA

Doutor

Universidade de Taubaté - Brasil edsonaaqo@gmail.com

MARCELA BARBOSA DE MORAES

Doutora

Universidade de Taubaté - Brasil marcela.moraes@unitau.com.br

RITA DE CÁSSIA RIGOTTI VILELA MONTEIRO

Doutora

Universidade de Taubaté - Brasil rita_rigotti@yahoo.com.br

Submetido em: 27/12/2015

Aprovado em: 20/11/2017

Doi: alcance.v24n4(Out/Dez).p495-512

\section{RESUMO}

A fim de manter a competitividade no mercado por meio de renovações em suas estratégias e carteira de produtos/serviços, as empresas investem cada vez mais na inovação. Essa pode ser compreendida como a criação de valor sustentável por meio de uma mudança de paradigma organizacional. Este trabalho tem como objetivo apresentar um ensaio teórico acerca das ferramentas de gerenciamento de projetos como fator de estímulo para o sucesso dos resultados em processos de inovação radical e/ou incremental. Objetiva-se também abordar a gestão de conhecimento como uma técnica de fomento à identificação e à idealização de produtos/serviços/processos e resultados inovadores, bem como se dá esse processo. Com esse fim, o estudo se utilizou de revisão bibliográfica para conceituar as características da inovação organizacional, bem como gestão de conhecimento e gerência de projetos. Sobre os mesmos, fez-se um comparativo entre as etapas de criação de produtos inovadores e a estrutura de criação de valor por projetos, que incorpora todas as suas fases desde a concepção estratégica até o retorno dos benefícios por ele gerados. Alocou-se também a posição estratégica dos principais roles (funções) de projetos de inovação. Na sequência, identificou-se em qual etapa de 
uma estratégia de inovação se dá início o ciclo de vida do projeto que resultará na mesma e, por fim, discutiu-se a importância da gestão de conhecimento como ferramenta de gestão que estimula o processo de identificação de oportunidades estratégicas e na idealização de novos projetos de características inovadoras. Como resultado, foi possível identificar que a utilização de técnicas e ferramentas de gestão de projetos são adequadas para o desenvolvimento de inovações radicais e incrementais, bem como confirmar a gestão de conhecimento como ferramenta de que estimula o processo de identificação de oportunidades estratégicas e na ideação de novos projetos com características inovadoras.

Palavras-chave: Gestão. Desenvolvimento. Inovação. Gerenciamento de Projetos. Gestão do Conhecimento.

\section{ABSTRACT}

In order to remain competitive in the market through renovations in their strategies and portfolio of products and services, companies are increasingly investing in innovation. This can be understood as the creation of sustainable value through a change in organizational paradigm. This work aims to present project management tools as factor for promoting a successful outcome in radical and/or incremental processes of innovation. It also addresses knowledge management as a development technique for the identification and idealization of products/services/processes and innovative results, and how this process occurs. A literature review was conducted to conceptualize the characteristics of organizational innovation, knowledge management and project management. In a second stage, a comparison was drawn between the stages of creating innovative products and projects and the structure of value creation of projects, which incorporates all the stages, from the strategic conception to the return of the benefits generated by it. The strategic position of the main roles (functions) of innovation projects is also allocated. Next, the study identifies the sage in which an innovation strategy starts off the life cycle of the project that will give rise to it and, finally, it discusses the importance of knowledge management as a management tool that encourages the process of identifying strategic opportunities and idealizing new innovative projects. As a result, it was identified that the use of project management techniques and tools is appropriate for developing radical and incremental innovations, as well as confirming the knowledge management as a tool that encourages strategic opportunities in the conception of new innovative projects.

Keywords: Management. Development. Innovation. Project Management. Knowledge Management.

\section{RESUMEN}

Con el fin de mantener la competitividad en el mercado por medio de renovaciones en sus estrategias y cartera de productos/servicios, las empresas invierten cada vez más en innovación. Eso puede ser comprendido como la creación de valor sostenible por medio de un cambio de paradigma organizacional. Este trabajo tiene como objetivo presentar un ensayo teórico acerca de las herramientas de gerenciamiento de proyectos como factor de estímulo para el éxito de los resultados en procesos de innovación radical e/o incremental. Se objetiva también abordar la gestión de conocimiento como una técnica de fomento a la identificación y a la idealización de productos/servicios/procesos y resultados innovadores, y como sucede este proceso. Con este fin, el estudio se basó en la revisión bibliográfica para conceptuar las características de la innovación organizacional y también gestión de conocimiento y gerencia de proyectos. Sobre los mismos, se hace una comparación entre las etapas de creación de productos innovadores y la estructura de creación de valor por proyectos, que incorpora todas las fases, desde la concepción estratégica hasta el retorno de los beneficios por él generados. Se designó también la posición estratégica de los principales roles (funciones) de proyectos de innovación. En la secuencia, se identificó en qué etapa de la estrategia de innovación se da el inicio al ciclo de vida del proyecto que resultará en la misma y, por fin, se discutió la importancia de la gestión de conocimiento como herramienta de gestión que estimula el proceso de identificación de oportunidades estratégicas y en la idealización de nuevos proyectos de curio innovador. Como resultado, fue posible identificar que la utilización de técnicas y herramientas de gestión de proyectos son adecuadas para el desarrollo de innovaciones radicales e incrementales, bien como confirmar la gestión de conocimiento como herramienta que estimula el proceso de identificación de oportunidades estratégicas y la idea de nuevos proyectos de curio innovador.

Palabras clave: Gestión. Desarrollo. Innovación. Gerenciamiento de Proyectos. Gestión de Conocimiento. 


\section{INTRODUÇÃO}

A fim de manter a competitividade no mercado pelas renovações em suas estratégias e carteira de produtos/serviços, as empresas têm investido cada vez mais na inovação. Essa pode ser compreendida como a criação de valor sustentável por meio de uma mudança de paradigma organizacional (CAVALCANTI et al., 2012). O PMI (2017) apresenta como alternativa para o sucesso no alcance de resultados exclusivos (únicos) as ferramentas e as técnicas de gerenciamento de projetos.

Esse trabalho tem como objetivo apresentar as ferramentas de gerenciamento de projetos como fator de estímulo para o sucesso dos resultados em processos de inovação radical e/ou incremental. Objetiva-se também apresentar a importância da gestão de conhecimento como técnica para identificação de oportunidades estratégicas e fomento à idealização de alternativas para criação de produtos/serviços/processos e resultados inovadores.

Com esse fim, dividiu-se o trabalho em cinco seções, sendo: a primeira dedicada à introdução do leitor ao tema da pesquisa; a segunda que apresenta os tópicos de revisão bibliográfica utilizados para conceituar as características de inovação, bem como gestão de conhecimento e gerência de projetos; a terceira seção apresenta o detalhamento do método utilizado para o alcance do proposto; a quarta seção dedica-se à discussão e aos resultados do estudo; e, por fim, a quinta seção apresenta as considerações finais do autor, bem como as referências bibliográficas utilizadas.

\section{REFERENCIAL TEÓRICO}

Esta seção do artigo apresentará o referencial teórico utilizado para o desenvolvimento do estudo. Discutirá o conceito de inovação, conceituará gestão de conhecimento e, por fim, gerência de projetos.

\subsection{Inovação}

A inovação pode ser definida como o processo de busca por renovação, embasada na soma de conhecimentos acumulados ao longo de um período de tempo e com fim em um bem inovador, sendo o mesmo tangível ou intangível (CORAZZA; FRACALANZA, 2004). Delimitando ao contexto empresarial, pode-se conceituar a inovação como a antecipação de criação de novos conceitos frente à concorrência (SIMANTOB; LIPPI, 2003).

Em outra definição, a inovação pode ser compreendida como a criação de valor sustentável por meio de uma mudança de paradigma interno da organização. Essa quebra de paradigma pode ser interpretada como, mas não se limita à comercialização de produtos/serviços, ampliação/desenvolvimento de vias de distribuição, elaboração de novos produtos/processos, aplicação de novas técnicas de marketing, entre outras (CAVALCANTI et al., 2012).

As maiores motivações para o ato de inovar são o estímulo para o desenvolvimento econômico e a criação de valor. Nesse interim, a inovação assume internamente nas organizações diversos papéis, podendo atuar em contextos diferenciados (SIMANTOB e LIPPI, 2003). A classificação dos tipos de inovação dá-se conforme 0 Quadro 1 do presente estudo.

Quadro 1 - Classificação dos tipos de inovação

\begin{tabular}{|l|l|}
\hline \multicolumn{1}{|c|}{ Classificação da inovação } & \multicolumn{1}{c|}{ Definição } \\
\hline Inovação de Produtos e Serviços & $\begin{array}{l}\text { Desenvolver e comercializar novos produtos e serviços, alicerçados em novos } \\
\text { conceitos tecnológicos e norteados pelas necessidades e satisfação dos clientes; }\end{array}$ \\
\hline Inovação de Processos & $\begin{array}{l}\text { Desenvolver novas formas de produção e/ou novas maneiras de relacionar-se com } \\
\text { o cliente para prestação de serviços; }\end{array}$ \\
\hline Inovação de Negócios & $\begin{array}{l}\text { Desenvolver novos modelos de negócio que forneçam vantagem competitiva de } \\
\text { forma sustentável; e }\end{array}$ \\
\hline Inovação de Gestão & Desenvolver novas estruturas hierárquicas. \\
\hline
\end{tabular}

Fonte: Adaptado de Sinantob e Lippi (2003). 
Independentemente do aspecto e da abrangência da inovação (sejam radicais ou incrementais), as mesmas acontecem fundamentalmente por duas razões: econômicas e tecnológicas. 0 Quadro 2 desse artigo elenca os principais motivos da opção pela inovação por organizações frente à razão da opção pelas mesmas.

Quadro 2 - Classificação dos tipos de inovação

\begin{tabular}{|c|c|}
\hline Razão da opção pela inovação & Descrição da inovação; \\
\hline \multirow{5}{*}{ Razões Tecnológicas } & Desenvolver novos produtos e serviços; \\
\hline & Alterar ou melhorar os métodos de produção existentes; \\
\hline & Imitar os líderes em inovação; \\
\hline & $\begin{array}{l}\text { Adaptar as tecnologias desenvolvidas por outros às necessidades da empresa; } \\
\mathrm{e}\end{array}$ \\
\hline & Melhorar o desempenho de técnicas existentes. \\
\hline \multirow{13}{*}{ Razões Econômicas } & Inovação de produtos: \\
\hline & - Substituir produtos obsoletos; \\
\hline & - $\quad$ Alargar a gama de produtos; \\
\hline & - Manter posição atual de mercado; e \\
\hline & - Entrar em/abrir novos mercados. \\
\hline & Inovação em processos: \\
\hline & - Melhorar a flexibilidade na fabricação; \\
\hline & - $\quad$ Reduzir custos de fabricação: \\
\hline & o Consumo de materiais; \\
\hline & ○ Consumo de Energia; e \\
\hline & o $\quad$ Taxa de defeitos de produção. \\
\hline & - $\quad$ melhorar condições de trabalho; e \\
\hline & - $\quad$ reduzir a poluição ambiental. \\
\hline
\end{tabular}

Fonte: Adaptado de Sinantob e Lippi (2003).

A IBM (2006) apresenta em seu estudo corporativo a inovação que o enfoque dos CEO's sobre esse cenário dá-se principalmente sobre: inovação de produtos, serviços e mercados; inovação sobre operações; e inovação sobre modelo de negócios. A alocação percentual de participantes, com cargo de alta gestão, da pesquisa realizada pela supracitada organização por classificação de inovação se dá conforme o gráfico presente na Figura 1 desse estudo. A mesma instituição reforça a importância do estímulo à inovação aos profissionais em cargos de liderança, visto que $35 \%$ das iniciativas inovadoras surgem nesse contexto (IBM, 2006). 
Figura 1 - Enfoque de CEO's por classificação de inovação

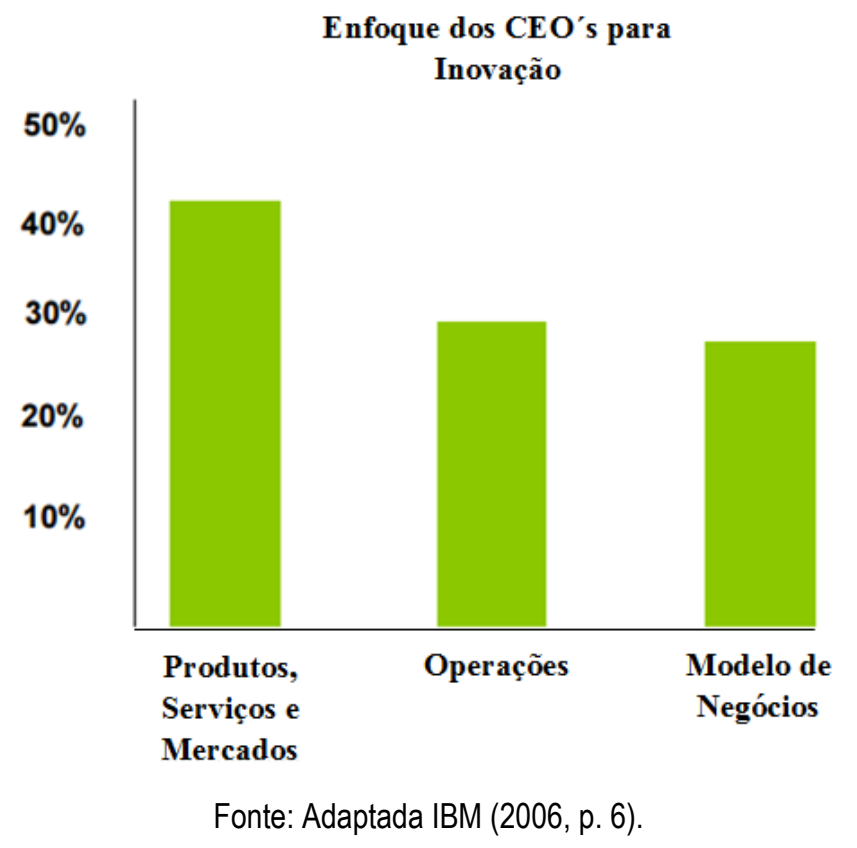

Em contraponto a essa colocação, De Bes e Kotler (2011) conceituam que no cenário empresarial a inovação como disciplina não atingiu um estágio de desenvolvimento capaz de sanar a necessidade de inovar, afirmando que muitas organizações ainda não possuem incentivos para criação de conceitos e, nas que visualizam retorno financeiro para 0 ato de inovar, a necessidade por inovação supera em muito a capacidade de fazê-lo. Os autores percebem também que a inovação deve ser compreendida amplamente, como o desenvolvimento de uma cultura inovadora dentro da corporação

Entretanto, a cultura da inovação somente alcançará seus objetivos se a organização assumir os ricos de alterar-se processos em funcionamento elou de ingressar em novas demandas de mercado (DE BES; KOTLER, 2011).

Segundo Sarkar (2010), uma das possíveis justificativas para o não investimento assíduo por parte das organizações na inovação em produtos e processos está na relutância em assumir os riscos inerentes a esse processo. $\mathrm{O}$ autor afirma que a disposição por correr riscos, bem como a tolerância a erros são indispensáveis para criação de um ambiente que propicie a inovação em organizações.

Simantob e Lippi (2003) complementam esse conceito quando afirmam que dentro das organizações sempre existirão limites para inovação, como prazo, orçamentos e demanda, entretanto é necessário vencer-se essas restrições aplicando-se a criatividade, a fim de inovar. Todavia o processo da inovação não necessariamente representa uma constante quebra de paradigma e que as inovações revolucionárias, chamadas de inovações radicais, são minoria frente às inovações de melhoria em produtos, serviços, processos operacionais e de gestão, chamadas de inovações incrementais (DE BES; KOTLER, 2011).

Drucker (2007) afirma que, independentemente da abrangência da inovação (radical ou incremental) e dos investimentos a ela destinados, o maior fator de sucesso em sua execução encontra-se na criatividade, nos conhecimentos, nas competências e na dedicação das pessoas intervenientes ao mesmo. De Bes e Kotler (2011) concordam com esse conceito e elencam as fontes significativas para o processo inovação, que podem ser visualizados na Figura 2. 
Figura 2 - Gráfico comparativo percentual das fontes significativas para o processo de inovação

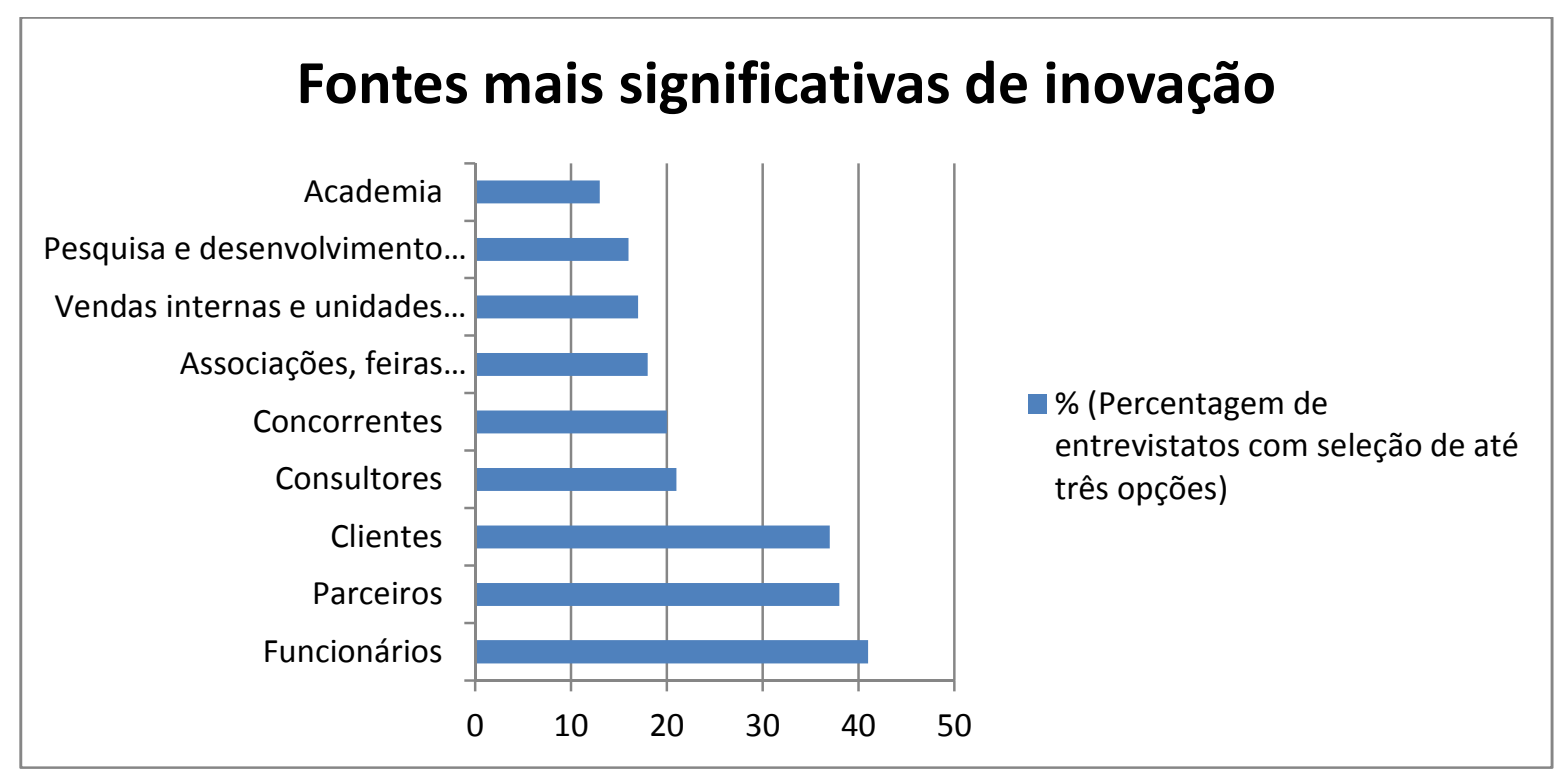

Fonte: Adaptada de De Bes e Kotler (2011).

Observa-se no gráfico presente na Figura 2 que, dentro do contexto empresarial, as competências em que se embasam os projetos de inovação são primordialmente dos funcionários e parceiros da organização. Como fator relevante, tem-se também a observação das necessidades do mercado a fim de prover produtos com algum grau de originalidade.

Com fim de sistematizar o processo contínuo da inovação organizacional de forma a estimular sua realização ininterrupta, De Bes e Kotler (2011) propõem o conceito A-F de inovação, em que determinam o papel de cada stakeholder dentro da criação de projetos inovadores para organização. Esse processo é dividido em seis etapas conhecidas como os "6ls da inovação". O Quadro 4 desse estudo apresenta o conceito de cada role (interveniente) do processo de inovação corporativa e suas respectivas fases de atuação.

Quadro 4 - Fontes significativas para o processo de inovação

\begin{tabular}{|l|l|c|}
\hline \multicolumn{1}{|c|}{ A-F } & \multicolumn{1}{|c|}{ Descrição } & Os 6 Is da inovação \\
\hline Ativadores & $\begin{array}{l}\text { Início do processo de inovação, podem influenciar membros do time } \\
\text { do projeto de inovação. }\end{array}$ & Iniciação \\
\hline Buscadores & $\begin{array}{l}\text { Especialistas em busca de informações pertinentes para iniciar gerar } \\
\text { novas ideias. }\end{array}$ & Informação \\
\hline Criadores & $\begin{array}{l}\text { Produtores de ideias, conceitos, possibilidades e novas soluções de } \\
\text { processo. }\end{array}$ & \multicolumn{1}{c|}{ Ideação } \\
\hline Desenvolvedores & $\begin{array}{l}\text { Especialistas em transformar ideias em produtos/ serviços tangíveis, } \\
\text { bem como elaborar o plano de marketing }\end{array}$ & Invenção \\
\hline Executores & $\begin{array}{l}\text { Responsáveis por lançar a inovação em desenvolvimento para uso } \\
\text { na organização ou no mercado }\end{array}$ & Implementação \\
\hline Facilitadores & $\begin{array}{l}\text { Aprovadores das despesas/investimentos necessários à medida que } \\
\text { o processo de inovação avança, impedindo sua paralisia. }\end{array}$ & Instrumentação \\
\hline
\end{tabular}

Fonte: Adaptado de De Bes e Kotler (2011).

A relação entre os intervenientes do processo de inovação dá-se conforme a representação da Figura 3. $O$ relacionamento entre os sujeitos do processo de inovação permite que as informações e o conhecimento sobre a iniciativa transitem para todos os envolvidos do núcleo de inovação, possibilitando uma visão ampla e um melhor aproveitamento das habilidades de profissionais de diversos segmentos, não somente do departamento de pesquisa e desenvolvimento, como ocorre em diversas organizações. 
Figura 3 - Relação entre os intervenientes do processo de inovação

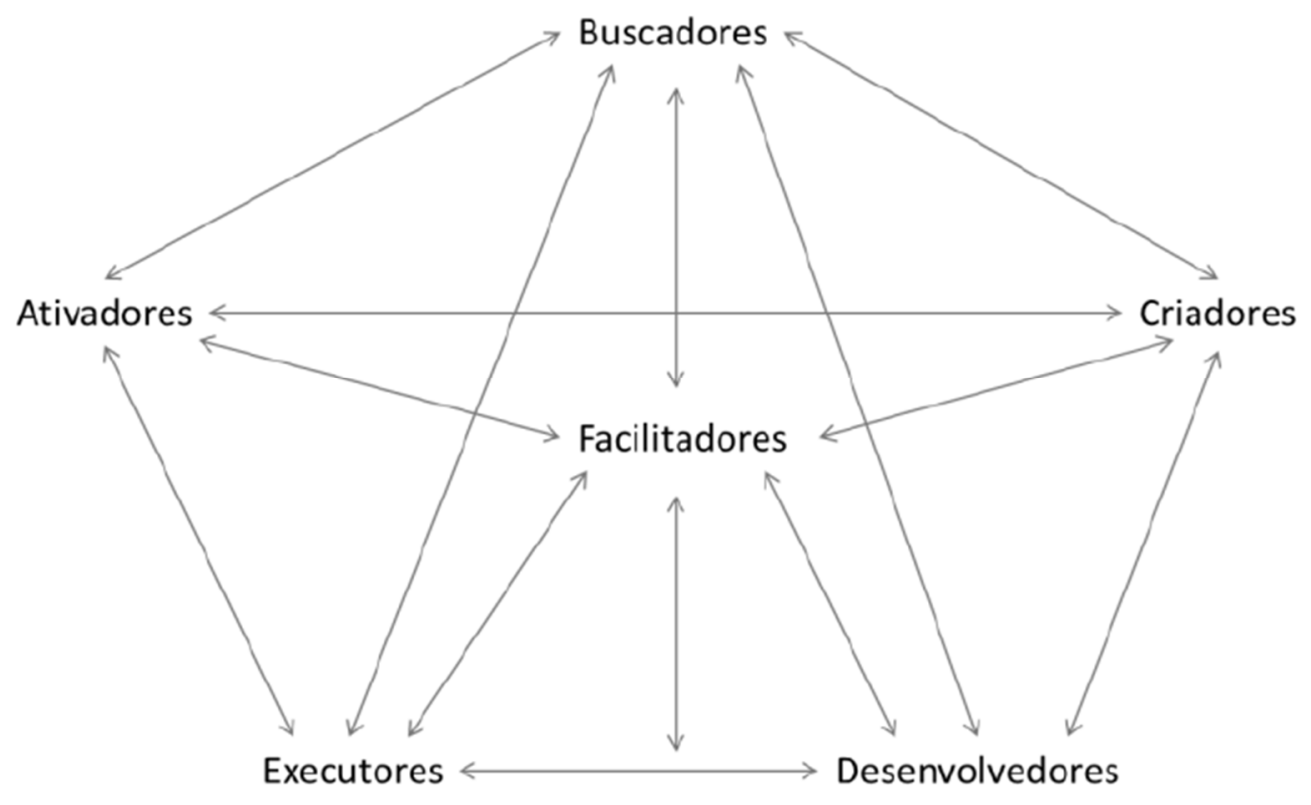

Fonte: Adaptada de De Bes e Kotler (2011).

Canongia et al. (2004) abordam que o processo da inovação muito depende da disseminação do conhecimento nos níveis organizacionais, a fim de que decisões departamentais fundamentadas nesse conhecimento possam estimular a criação de bens e processos inovadores em todas as áreas da empresa. Com esse enfoque e com o fim de possibilitar a discussão sobre a importância desse conhecimento proveniente dos projetos de inovação, o próximo tópico dessa revisão tratará a gestão do conhecimento.

\subsection{Gestão de Conhecimento}

$\mathrm{Na}$ analogia proposta por Morgan (2000) as organizações podem ser comparadas a cérebros devido a administrarem um grande número de dados e informações compilando-os e processando-os em sistemas informatizados para, posteriormente, pela análise inteligente de pessoas, serem transformados em conhecimento.

Num ambiente de negócios, pode considerar-se "dado" todo registro puro e simples de eventos organizacionais ou textos estruturais de transações realizadas. No mesmo cenário, a "informação" pode ser definida como o resultado da análise dos dados com o propósito de modificar a percepção, o julgamento e/ou a atitude de seu receptor sobre o contexto sobre a qual a mesma intervém (LINS, 2003).

Com fim de gerar informações sobre uma dada massa de dados, Davenport e Prusak (1998) definem a aplicação das técnicas:

- Contextualização: identificação da finalidade dos dados coletados;

- Categorização: identificação dos componentes essenciais dos dados;

- Cálculo: análise estatística sobre os dados;

- Correlação: mitigação dos erros em dados; e

- Condensação: apresentação dos dados de forma concisa.

Conhecimento pode ser definido como uma crença justificadamente verdadeira (TAKEUCHI; NONAKA, 2008). De maneira mais completa, Davenport e Prusak (1998) apresentam o conhecimento como um amálgama fluido de experiências condensadas, valores, informações contextuais e insights, permitindo a avaliação e a 
incorporação de novas experiências. Dentro das organizações o mesmo pode estar incorporado a documentos, rotinas operacionais, processos, práticas e normas organizacionais. Ideia complementada por Friesl (2012), que conceitua o conhecimento organizacional como um bem que pode ser construído socialmente por indivíduos dentro de uma organização, enquanto se envolvem em suas atividades diárias para atingir objetivos predefinidos.

Segundo Takeuchi e Nonaka (2008), o conhecimento pode ser classificado em: conhecimento tácito e conhecimento explícito. Nessa abordagem o conhecimento tácito é definido como pessoal e específico ao contexto do qual faz parte, sendo dessa forma de difícil formalização e transmissão.

Em contraponto, o conhecimento explícito, ou codificado, refere-se ao conhecimento sistematizado, ou seja, transmissível em linguagem formal. Esse pode ser expresso em texto e número, sendo de fácil comunicação e compartilhamento. Podem ser expressos, como exemplo, em: fórmulas científicas, princípios universais, especificações técnicas e manuais.

O conhecimento institucional é gerado pela transformação do conhecimento tácito em explícito e viceversa, e elencam como fases do processo de conversão do conhecimento os modos de conversão apresentados no Quadro 5 (TAKEUCHI; NONAKA, 2008).

Quadro 5 - Fases de conversão de conhecimento tácito em explícito.

\begin{tabular}{|l|l|l|l|}
\hline $\begin{array}{c}\text { Modos de conversão } \\
\text { do conhecimento: }\end{array}$ & \multicolumn{1}{|c|}{$\begin{array}{c}\text { Descrição dos modos de } \\
\text { conversão do conhecimento: }\end{array}$} & $\begin{array}{l}\text { Processo de conversão } \\
\text { do conhecimento } \\
\text { de/para: }\end{array}$ & \multicolumn{1}{|c|}{$\begin{array}{c}\text { Ocorrência entre os } \\
\text { intervenientes: }\end{array}$} \\
\hline Socialização & $\begin{array}{l}\text { Compartilhar e/ou criar } \\
\text { conhecimento tácito por meio de } \\
\text { experiências diretas; }\end{array}$ & $\begin{array}{l}\text { De conhecimento tácito } \\
\text { para conhecimento tácito }\end{array}$ & De indivíduo para indivíduo \\
\hline Externalização & $\begin{array}{l}\text { Externalização: Articular } \\
\text { conhecimento tácito pelo diálogo } \\
\text { e reflexão; }\end{array}$ & $\begin{array}{l}\text { De conhecimento tácito } \\
\text { para conhecimento } \\
\text { explícito }\end{array}$ & De indivíduo para grupo \\
\hline Combinação & $\begin{array}{l}\text { Combinação: Sistematizar e } \\
\text { aplicar o conhecimento explícito e } \\
\text { a informação; e }\end{array}$ & $\begin{array}{l}\text { De conhecimento explícito } \\
\text { para conhecimento } \\
\text { explícito }\end{array}$ & Do grupo para organização \\
\hline Internalização & $\begin{array}{l}\text { Internalização: Aprender e } \\
\text { adquirir novo conhecimento tácito } \\
\text { na prática. }\end{array}$ & $\begin{array}{l}\text { De conhecimento explícito } \\
\text { para conhecimento tácito }\end{array}$ & $\begin{array}{l}\text { Da organização para o } \\
\text { indivíduo }\end{array}$ \\
\hline
\end{tabular}

Fonte: Adaptado de Takeuchi e Nonaka (2008).

Sobre esses quatro pilares, a transformação de dados em conhecimento socializa o conhecimento individual em toda organização. Nesse contexto, cada indivíduo torna-se único e capaz, por meio de seus conhecimentos, habilidades e competências, contribuir para o grupo e para organização como um todo (FRANCO et al., 2013).

Ao reconhecer-se a soma de conhecimentos individuais como bem organizacional, proporcionando-lhe vantagem competitiva, cria-se na corporação o conceito de capital intelectual (STEWART, 1998). Sita et al. (2013) expõem como necessidade primária para organizações a retenção de seu capital intelectual, e afirmam que cada indivíduo que deixa a organização leva consigo parte desse capital. Ressaltam também a importância de se criar uma cultura e mecanismos para retenção dessas informações e experiências vivenciadas pelos colaboradores da empresa, tornando-a mais competente e produtiva.

Alvarenga e Drummond (2005) apresentam como principais motivadores para a adoção da gestão do conhecimento pelas organizações: 1 - reconhecimento da informação como importante fator de competitividade comercial; 2 - necessidade de inovação contínua; 3 - problemas relativos à disseminação de informações, evidenciando a falta de gestão das mesmas; 4 - falta de rotinas para compartilhamento e proteção das informações; 5 - mudança cultural para cultura colaborativa; e 6 - estimular a aprendizagem organizacional.

Luo et al. (2012) conceituam que a aquisição de conhecimento pode ser vista como o processo de identificá-lo e engajá-lo por ações diretas e indiretas, por meio de colaboração, consultorias, interação com 
especialistas na área de conhecimento, formação e outras atividades com fim de identificar novas fontes de conhecimento.

Takeuchi e Nonaka (2008) apresentam cinco fatores para promoção da criação do capital intelectual da organização:

- Incutir a visão de conhecimento: comunicar a necessidade de transferir a mecânica da estratégia de negócios a fim de se criar uma visão geral do conhecimento organizacional. Essas informações, se corretamente gerenciadas, proverão ao plano estratégico de negócios, por meio do pleno conhecimento de suas vantagens competitivas, uma sólida estratégia de avanço;

- Gestão das conversações: gerenciar as comunicações entre colaboradores que carregam a essência das atividades organizacionais;

- Mobilização de ativistas do conhecimento: correta alocação de profissionais que estimulam e aproveitam o conhecimento organizacional preestabelecido e criam nas atividades executadas novos conhecimentos. Esses colaboradores devem ser utilizados pela organização com seis propósitos: foco e inicialização da criação do conhecimento; redução do tempo e custos necessários para criação do conhecimento; alavancagem de iniciativas para criação do conhecimento; melhora das condições para criação do conhecimento; preparação de profissionais participantes do contexto de criação do conhecimento para novas tarefas; e estímulo da discussão em grupo de fatores de transformação organizacional;

- Criação do contexto correto: verificação da relação entre a estrutura organizacional, a estratégia e a promoção do conhecimento, a fim de garantir que a empresa desenvolva as condições adequadas para estimular a criação de conhecimento institucional;

- Globalização do conhecimento: garantir que a informação gerada em uma unidade local atinja de maneira rápida e eficiente as demais unidades da corporação, levando-se em consideração as particularidades regionais que devem ser analisadas e adaptadas frente ao cenário enfrentado por cada unidade específica.

Para Sita et al. (2013), um programa eficiente de gestão do capital intelectual deve considerar para além de contingências, a fim de reter o capital intelectual intrínseco aos colaboradores, por meio de medidas de reconhecimento e recompensa o apoio da tecnologia da informação, a fim de armazenar de forma normatizada a grande massa de dados gerada com a implantação de um programa de gestão do conhecimento, bem como disponibilizar essas informações quando necessárias para uso.

Terra (2005) afirma que, no Brasil, verifica-se o aumento da importância da gestão de conhecimento empresarial devido aos desafios impostos pela globalização, obrigando as organizações à tomada de ações estratégicas nesse âmbito, a fim de manter sua competitividade internacional.

\subsection{Gerência de Projetos de Inovação}

A execução de projetos não é uma prática nova, desde a antiguidade gerenciam-se projetos, como a construção de templos, pirâmides, caravelas, entre outros. Entretanto, atualmente recursos financeiros, de tempo e humanos, têm grande relevância, tornando-se necessária a incorporação de métodos para seu gerenciamento de forma a reduzir o desperdício desses recursos e concluir o empreendimento dentro dos parâmetros de prazo e custo previstos (KEELING; BRANCO, 2012).

Balashov (2008) afirma que a gerência de projetos de inovação pode ser considerada a definição de objetivos, planejamento das atividades e acompanhamento de sua aplicação, com apoio da estrutura organizacional, centrados na implementação de uma ideia inovadora. Já Khodyreva (2015) conceitua a gestão de projetos de inovação como a arte de administrar os recursos humanos e materiais ao longo do ciclo de vida do projeto, utilizando-se de métodos e técnicas para alcançar resultados específicos, considerando seu custo, duração, qualidade e satisfação de seus participantes.

A Associação Brasileira de Normas Técnicas ressalta na norma ABNT ISO 21500 (2012) que o ambiente de projetos empreendidos por uma companhia é distinto do ambiente de operações realizadas por ela diariamente. 
A mesma instituição reforça a característica de singularidade dos projetos, afirmando que, embora existam similaridades entre eles, cada projeto é único, podendo suas diferenças ser caracterizadas nas entregas fornecidas; na influência exercida pelas partes interessadas; nos recursos utilizados; nas premissas; nas restrições; ou no modo como os processos são adaptados para gerar as entregas (ABTN, 2012).

Keeling e Branco (2012) afirmam que o encerramento do projeto se dá quando:

- as metas determinadas são alcançadas;

- $\quad$ por decisão o projeto torna-se inviável; ou

- $\quad$ as necessidades que motivaram sua criação não existem mais.

As empresas normalmente estabelecem estratégias de mercado baseadas em suas missões, visões, políticas e fatores fora dos limites organizacionais (ABNT, 2012), e os projetos são comumente utilizados para atingir os objetivos traçados nos planos estratégicos, e são autorizados para: alcançar uma nova demanda de mercado; aproveitar uma oportunidade de negócios; atender a solicitação de um cliente; desenvolver uma nova tecnologia; e atender a uma legislação, entre outros (PMI, 2017).

A Figura 5 ilustra a estrutura de criação de valor estratégico de projetos.

Figura 5 - Estrutura de criação de valor

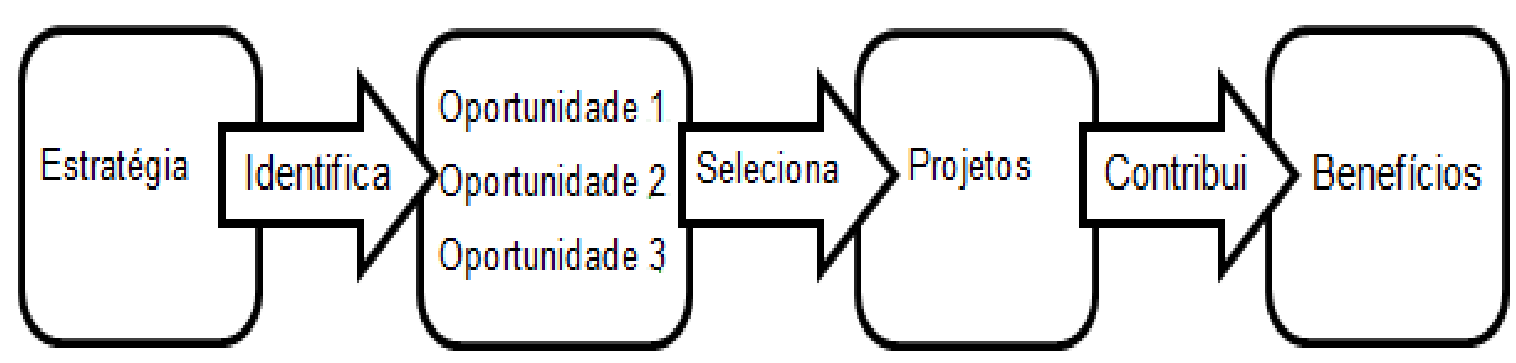

Fonte: Adaptada da ABTN (2017, p. 5).

Os projetos podem ser desenvolvidos em todos os níveis da organização, envolvendo uma pequena quantidade de pessoas ou milhares delas, e por muitas vezes ultrapassam os limites da organização, envolvendo fornecedores, parceiros, clientes e governo (VARGAS, 2017).

O gerenciamento desses empreendimentos (projetos) pode ser definido como a aplicação de métodos, ferramentas, técnicas e competências para garantir o alcance das metas estipuladas (ABTN, 2012).

Keeling e Branco (2012) elencam como principais papéis do gerente de projetos:

- ser responsável pelo bom desempenho do projeto;

- ser o principal agente para realização da integração de informações das diferentes frentes do projeto;

- ser o elo entre os intervenientes internos e externos, e as organizações;

- ser regulador do progresso, velocidade, qualidade e custo do projeto;

- ser o líder motivador dos recursos humanos do projeto;

- ser o comunicador e negociador em todas as interações do projeto; e

- ser o administrador das finanças e demais recursos do projeto.

Vargas (2017) reforça, entretanto, que muito do sucesso do gerente de projetos depende do favorecimento do ambiente de projetos pela organização. 
A norma 21500 da ABTN (2012) reforça a necessidade de análise do ambiente de projetos pelo gerente e pela sua equipe, afirmando que esse time deve considerar fatores externos à organização: socioeconômicos, geográficos, políticos, de regulamentações, tecnológicos e ecológicos. Descreve, ainda, os fatores internos que podem influenciar no gerenciamento do projeto, tais como estratégia, tecnologia, maturidade de gerenciamento, disponibilidade de recursos, cultura organizacional e estrutura da empresa.

O Instituto PMI (2012) aponta como boa prática para a gerência do projeto a sua divisão do mesmo em quarenta e sete processos distintos, distribuídos em dez áreas de conhecimento: escopo, tempo, custo, qualidade, aquisições, risco, comunicação, recursos humanos, partes interessadas e integração.

Porém a mesma instituição ressalta que esses processos não devem ser aplicados de forma uniforme em todos os projetos, e que cabe ao gerente de projetos juntamente à sua equipe definir quais processos são apropriados para um projeto específico e em que grau de rigor deve ser utilizado (PMl, 2017).

Os processos estão agrupados em grupos, e envolvem cinco fases integradas:

- iniciação: fase que formaliza a existência do projeto para a organização; estipula seus objetivos e seu escopo inicial; nomeia o gerente de projeto; e autoriza a disponibilização de recursos da empresa para sua realização;

- planejamento: grupo de processos com finalidade de determinar, com melhor grau de precisão, 0 que deve ser feito, com a declaração de escopo, e como deve ser feito, pelo plano de gerenciamento de projeto. As definições serão registradas em uma linha de base, plano contra o qual os resultados serão conferidos;

- execução: processos com finalidade de prover a produção das entregas do projeto por meio da integração de pessoas, organizações e recursos materiais;

- monitoramento e controle: fase de conferência de resultados da execução, comparando-a com a linha de base definida no planejamento para que, no caso de desvios, ações sejam tomadas; e

- encerramento: etapa que formaliza o término do projeto, 0 aceite dos resultados obtidos, 0 encerramento oficial de contratos e a desmobilização da equipe (VALLE et al., 2007).

A relação administrativa entre os grupos de processos se dá conforme a Figura 6.

Figura 6 - Relação dos grupos de processo nas fases do projeto

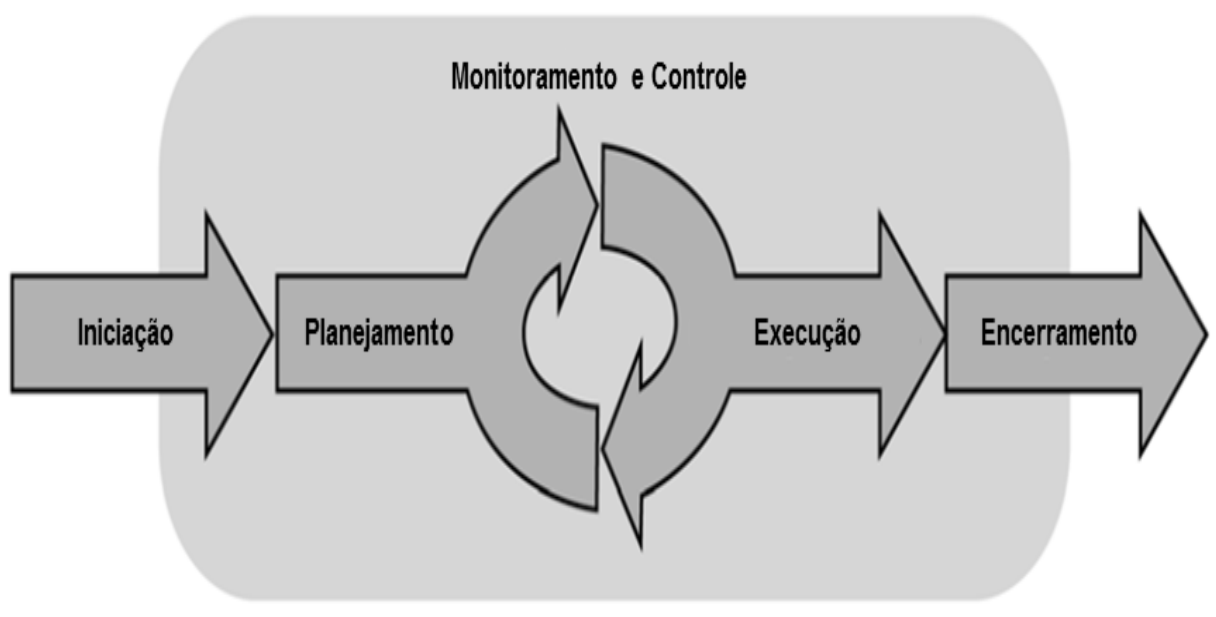

Fonte: Adaptada do PMI (2017, p. 19).

Observa-se, nessa estrutura, que após o início do projeto, realiza-se o planejamento das demais fases. Há uma comunicação direta com a execução de modo a permitir ajustes no plano, caso ocorram situações em que mudanças se façam necessárias. 
A essa relação dá-se o nome de planejamento em ondas sucessivas. Quando o produto ou resultado da entrega está concluído, inicia-se a fase de encerramento com a entrega e o aceite do cliente.

Os processos de monitoramento e controle acompanham todo o ciclo de vida do projeto, possibilitando ao gerente antecipar suas decisões aos possíveis riscos iminentes que venham a surgir.

A estrutura apresentada na Figura 6 deve, conforme o descrito no PMBOK versão 5 (PMI, 2017), ser utilizada para cada entrega do projeto de forma sequencial: início de um novo ciclo ao término do antecessor; ou sobreposta: a nova fase inicia-se antes do término da anterior. É papel do gerente do projeto administrar essas iterações de modo a garantir que seja entregue conforme o planejado (VARGAS, 2017).

Prado (2010) afirma que um dos fatores de sucesso para o gerenciamento de projetos é o amadurecimento da organização nas práticas de gerência de projetos, que propicia uma maior aderência dos custos e dos resultados entregues à linha de base definida na fase de planejamento.

\section{MÉTODO}

Meneghetti (2011) conceitua um ensaio teórico como a construção de diferentes percepções ou ideias acerca de um determinado tema sob a ótica do autor.

Esse ensaio teórico tem o objetivo de discutir a abordagem de gerenciamento de projetos para obtenção de produtos, serviços, processos e/ou resultados inéditos ou aperfeiçoamentos realizados sobre produtos/processos/serviço/ resultados existentes, bem como analisar o papel da gestão de conhecimento organizacional como fator de estímulo ao sucesso de projetos com características inovadoras.

A abordagem metodológica para realização da mesma utilizou-se de fontes bibliográficas para 0 levantamento dos conceitos de inovação, gestão de conhecimento e gerência de projetos, indispensáveis para proposta discussão.

Em posse do estado da arte literário sobre os supracitados temas, realizou-se um comparativo entre as fases de criação de produtos inovadores propostos por De Bes e Kotler (2011) e a estrutura de criação de valor por projetos proposta pela ABNT (2012). Alocou-se também a posição estratégica dos principais roles (funções) de projetos de inovação.

Dando sequência, analisou-se em qual etapa de uma estratégia de inovação inicia-se as etapas do ciclo de vida do projeto da originará (PMI, 2017). E, por fim, discorreu-se sobre a importância da gestão de conhecimento como ferramenta que estimula o processo de identificação de oportunidades estratégicas e de idealização de novos projetos que têm como resultados produtos, serviços, processos e/ou resultados inovadores, bem como a aplicação do modelo SECI (TAKEUCHI; NONAKA, 2008) nos projetos de inovação.

\section{RESULTADOS E DISCUSSÃO}

Projetos são empreendimentos executados em períodos de tempo predeterminados, planejados sucessivamente (ao longo de seu ciclo de vida) e com fim de gerar resultados exclusivos (PMl, 2017). Essa característica é bastante apropriada para a ação de criar-se bens, serviços, processos e resultados inovadores. Com os adventos da temporariedade, que confere ao projeto um término predefinido, e o planejamento ao longo de todo, o ciclo de vida que reduz consideravelmente o risco de fracasso do empreendimento, pode-se considerar que as ferramentas e as técnicas da gestão de projetos são as mais eficientes para condução de um projeto com fim inovador.

Entretanto, conforme o PMI (2017), todo projeto é empreendido em um ambiente muito mais amplo que o próprio projeto, e o entendimento de contexto inicia-se com a análise do ambiente externo e interno à organização, sobre os quais serão traçadas as estratégias organizacionais, base para a estrutura de criação de valor por projetos.

Nos projetos de inovação, a necessidade de sólidas estratégias torna-se ainda mais crítica, pois a geração de inovação radical ou incremental resulta em um produto, serviço, processo e/ou resultado de natureza inédita, cujo risco da não compreensão do conceito, ou não aceitação de seu resultado, podem acarretar o fracasso do empreendimento e, assim, em prejuízos financeiros e, por vezes, de imagem da instituição. 
Para uma melhor compreensão de como a estrutura de criação de valor por projetos sobrepõe as fases propostas por De Bes e Kotler (2011) para criação de produtos inovadores, conhecidas como "6 is da inovação", foi elaborado para o presente estudo o fluxo apresentado na Figura 7 desse artigo.

Figura 7 - Relação entre a cadeia de valor de projetos e fases do processo de inovação

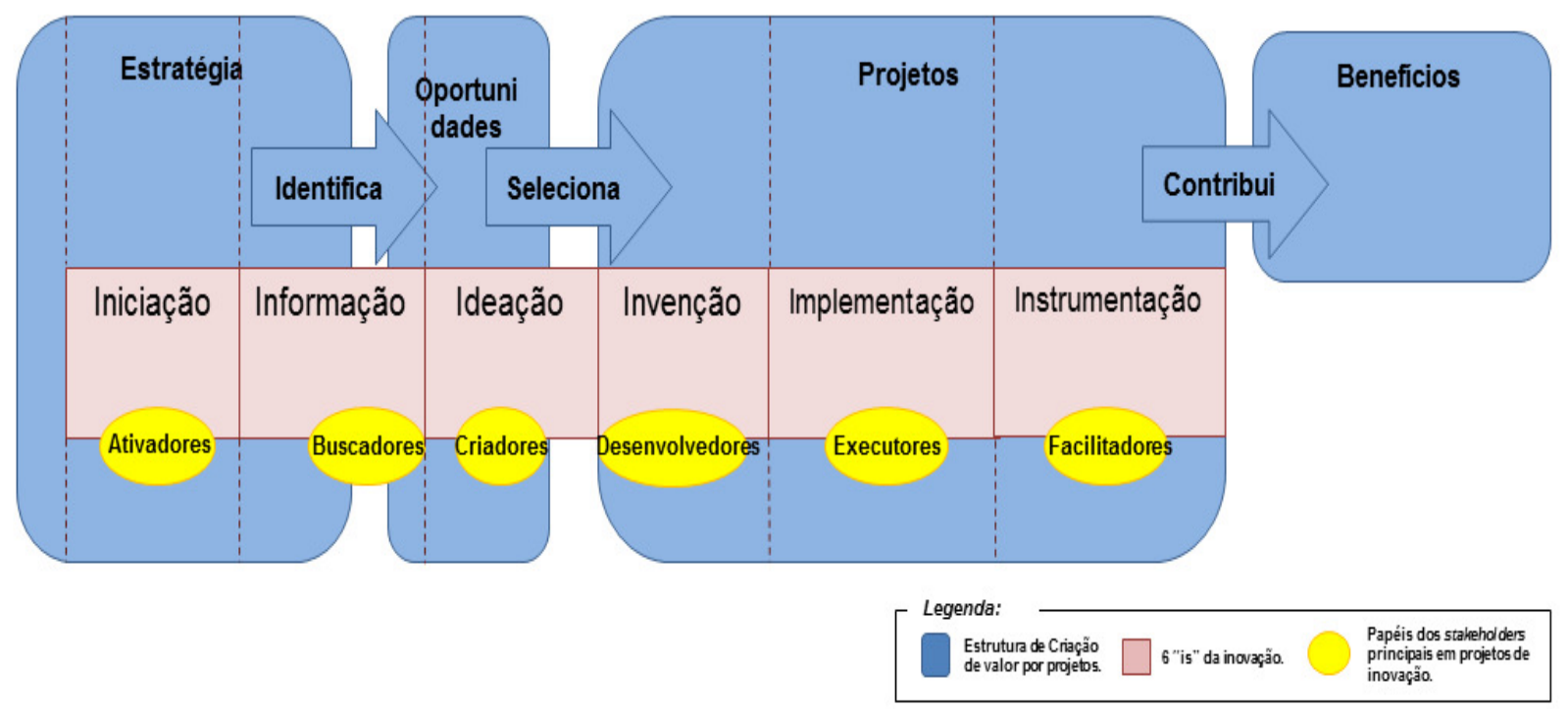

Fonte: Elaborada pelos autores.

Esse fluxo demonstra que a fase de iniciação, etapa em que se dá o primeiro estímulo para iniciativas inovadoras, está alocada dentro da estratégia organizacional. Pelo levantamento de informações do ambiente externo, identificam-se oportunidades para criação de produtos inéditos ou incrementos em produtos/processos existentes, a fim de manter-se a competitividade de mercado.

O levantamento das oportunidades possibilita então a fase de ideação do processo inovativo, em que se define sobre a estratégia da empresa, quais as prioridades da instituição no quesito de inovação de produtos, serviços, processos e/ou resultados.

Inicia-se então o desenvolvimento dos projetos para alcance das estabelecidas metas. 0 mesmo deve contemplar projetos com fim inovador, as etapas de invenção, implementação e instrumentação, abrangendo o período desde a criação da proposta inovação, até seu lançamento no mercado, incluindo todo trabalho necessário para sua compreensão por parte do público-alvo. Essa aceitação, por fim, resulta nos benefícios almejados pela organização.

Observa-se também a ação dos intervenientes principais do processo de inovação dentro do contexto da criação de valor por projetos, cujos ativadores atuam no nível estratégico; buscadores entre o nível estratégico e, juntamente com criadores no nível das oportunidades; e desenvolvedores, executores e facilitadores executam suas atividades na etapa dos projetos.

Entretanto, visto que a definição do produto a ser entregue pelo projeto encontra-se na fase de iniciação do mesmo, exclusivamente para projetos de características inovadoras, o ciclo de vida deve iniciar-se antecipadamente frente aos demais, pois a etapa da criação, em que se definem as características da inovação proposta, encontra-se na fase de oportunidades, sob atribuição dos criadores.

Dessa forma, agregando-se o ciclo de vida do projeto ao comparativo da estrutura de criação de valor frente às fases de criação de produtos inovadores, tem-se como resultado a representação na Figura 8. 
Figura 8 - Relação entre a cadeia de valor de projetos de inovação e o ciclo de vida dos projetos

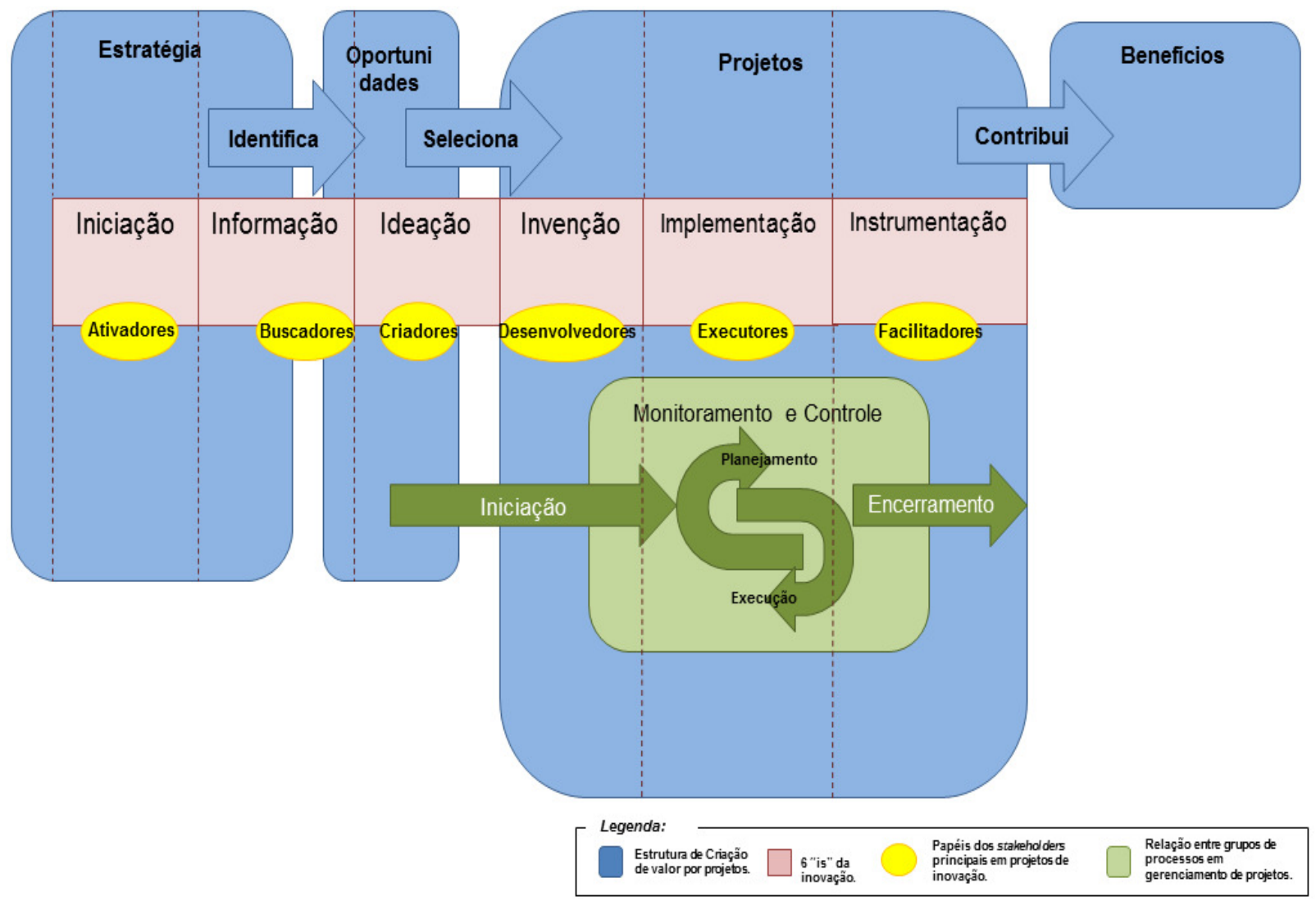

Fonte: Elaborada pelos autores.

Observa-se que, devido à natureza dos projetos de inovação, os stakeholders (partes interessadas) devem compreender criteriosamente o que será realizado, o resultado a ser alcançado e os riscos positivos e negativos da inovação, que a fase inicial do projeto deve estender-se para propiciar e formalizar todo esse contexto, em especial aos patrocinadores, com objetivo de obter sua aprovação e adquirir recursos para realização do projeto.

Entretanto, para uma correta contextualização da inovação e do proposto projeto para seu desenvolvimento, é necessária uma fundamentação da análise do contexto sobre informações estratégicas armazenadas e gerenciadas pela organização. Essa análise aumentará as chances de que o projeto resulte nos benefícios esperados pela organização.

Essas informações devem advir do conhecimento da organização sobre seu ambiente externo, as quais são representadas, mas não se limitam à percepção sobre demanda de aceitação do novo produto/serviço/processo/resultado ou de suas novas características pelo público-alvo estipulado, tempo de reação da concorrência, impactos por quebra de paradigma, entre outros.

Outra fonte não menos importante de informações é o conhecimento organizacional proveniente da cultura institucional, conhecimento das forças da organização, do histórico de lições aprendidas pela realização de projetos anteriores, entre outros.

Apesar de ser considerada uma rica fonte de informações que fundamentarão e assegurarão os resultados dos projetos de inovação empreendidos, bem como sua prioridade de execução, o conhecimento organizacional somente será considerado maduro se houver internamente na organização padrões e ferramentas para sua adequada formalização e armazenamento.

Os supracitados processos e ferramentas devem abranger todo conhecimento adquirido pela análise do ambiente externo, bem como interno da organização. No caso de projetos, é recomendável que o conhecimento proveniente das informações de acertos e erros produto de sua execução, dos benefícios gerados pelo resultado 
do mesmo, bem como todo conhecimento tácito e explícito advindo dos stakeholder (intervenientes) seja atualizado nos autos de lições aprendidas.

Com maior criticidade, as lições aprendidas durante os projetos de inovação, bem como os benefícios alcançados por sua execução, devem incorporar-se de maneira sistematizada à base de conhecimentos organizacionais, pois além de auxiliarem em decisões estratégicas para futuros projetos, também serão uma importante referência para futuras inovações incrementais sobre o resultado do projeto empreendido.

A gestão do conhecimento em projetos de inovação incorpora-se frente aos demais contextos de projetos com foco em inovação estudados nesse artigo, conforme a Figura 9.

Figura 9 - Relação entre a cadeia de valor de projetos de inovação e gestão do conhecimento

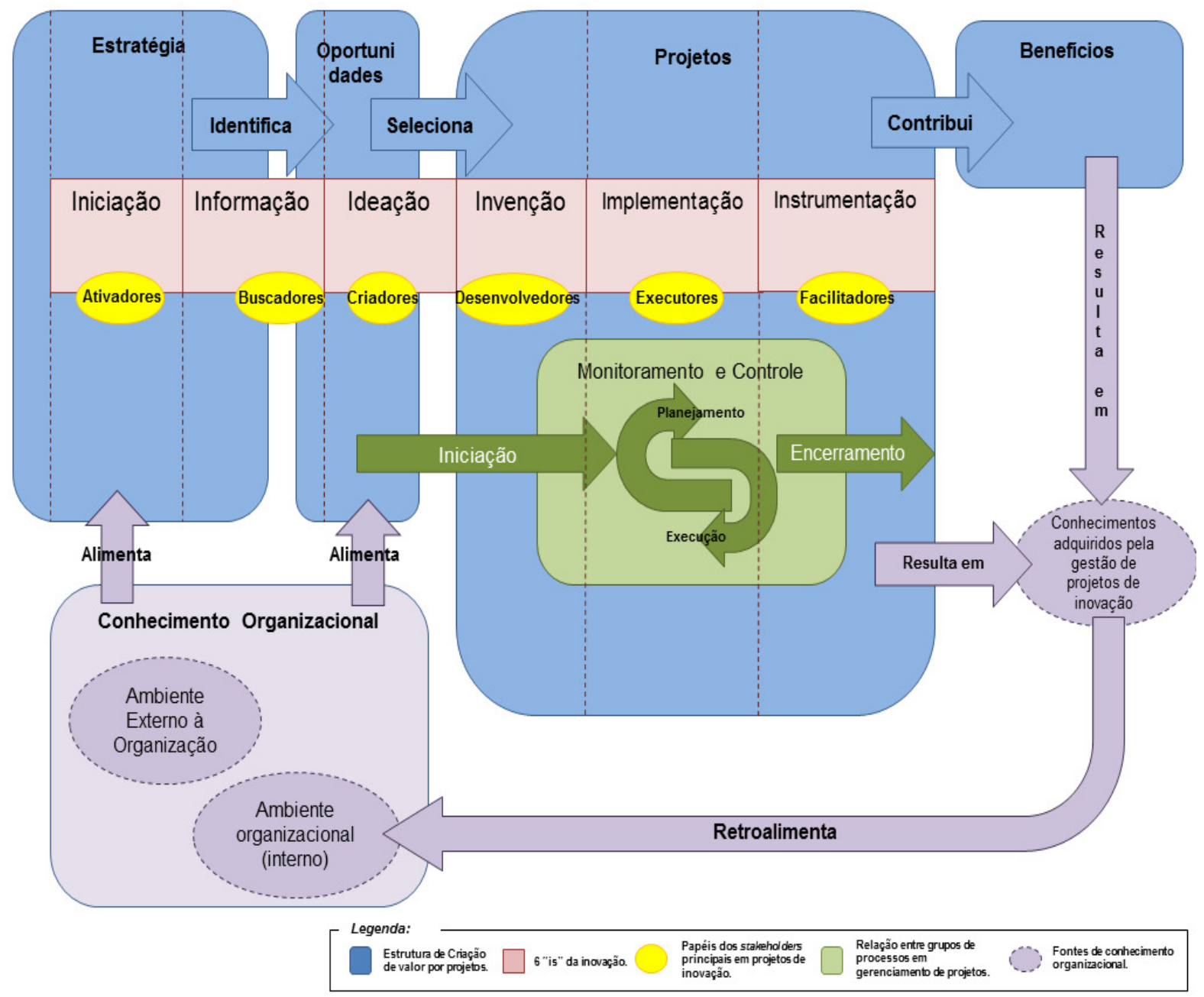

Fonte: Elaborada pelos autores.

A representação gráfica constante na Figura 9 contempla todo o cenário pesquisado para elaboração desse estudo, abrangendo a relação entre a estrutura de criação de valor por projeto, as fases e principais funções do processo de inovação, o ciclo de vida do projeto e, por fim, o conhecimento proveniente da execução de projetos de inovação e sua influência para identificação de novas abordagens estratégicas e oportunidades para criação de inovações radicais ou renovações em produtos já consolidados pela companhia.

Incorporando-se as fases do modelo SECI (Socialização, Externalização, Combinação e Internalização) para uma melhor compreensão das conversões de conhecimento tácito em explicito, tem-se que a análise da estratégia e das oportunidades deve ocorrer pela internalização do conhecimento sistematizado pela companhia. 0 mesmo deve ser, ao longo da fase de projetos, disseminado e socializado de modo a condicionar o trabalho 
ao previamente aprendido, e evoluído por meio das novas experiências vivenciadas durante a execução do projeto empreendido.

As novas experiências devem, por sua vez, também ser sistematizadas (externalizadas) e incorporadas à base de conhecimento da organização (combinação), servindo posteriormente de base para novas decisões estratégicas e execuções de projetos.

Conforme o proposto no modelo $\mathrm{SECl}$, esse ciclo de aprendizado é contínuo. Dando fim à seção de discussão, apresenta-se a representação gráfica do modelo SECl sobre a estrutura de cadeia de valor de projetos de inovação, conforme a Figura 10.

Figura 10 - Relação entre a cadeia de valor de projetos de inovação e o modelo SECl

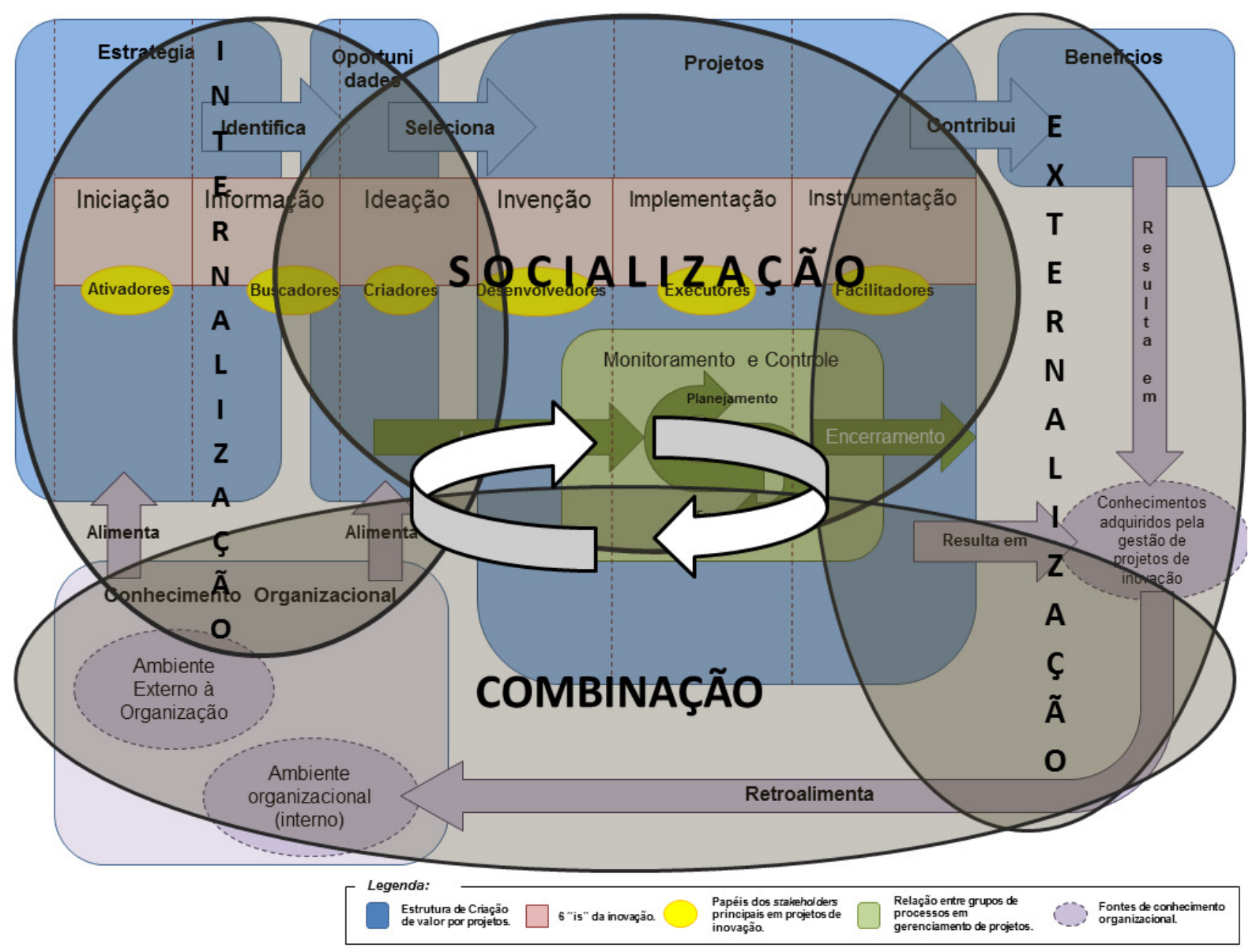

Fonte: Elaborada pelos autores.

A próxima seção desse artigo o encerra, apresentando as considerações finais do autor e as referências bibliográficas por ele utilizadas para elaboração do mesmo.

\section{CONSIDERAÇÕES FINAIS}

Este trabalho buscou apresentar um estudo teórico acerca das ferramentas de gerenciamento de projetos como fator de estímulo para o sucesso dos resultados em processos de inovação radical e/ou incremental. Objetivou-se também abordar a gestão de conhecimento como uma técnica de fomento à identificação e à idealização de produtos/serviços/processos e resultados inovadores, bem como esse processo ocorre. 
Dentro do contexto da inovação, buscou-se com a realização desse estudo discutir a abordagem de gerenciamento de projetos para obtenção de produtos, serviços, processos e/ou resultados inéditos ou, ainda, aperfeiçoamentos realizados sobre produtos, serviços, processos e/ou resultados existentes.

Analisou-se também o papel da gestão de conhecimento organizacional como fator de estímulo ao sucesso em projetos com características inovadoras.

Para o alcance dos objetivos propostos, realizou-se inicialmente um comparativo entre as fases de criação de produtos inovadores propostos por De Bes e Kotler (2011) no modelo A-F e a estrutura de criação de valor por projetos, que incorpora todas as suas fases, desde a concepção estratégica até o retorno dos benefícios por ele gerados. Alocou-se também nesse interim a posição estratégica dos principais roles (funções) de projetos de inovação.

Na segunda fase de desenvolvimento da discussão proposta, buscou-se identificar em qual etapa de uma estratégia de inovação se iniciaria o ciclo de vida do projeto que resultará na mesma.

Verificou-se por meio dessa análise e da similaridade de conceitos, como a exclusividade de resultados e planejamento progressivo, que a utilização de técnicas e ferramentas de gestão de projetos são adequadas para o desenvolvimento tanto de inovações radicais quanto de incrementais.

Por fim, discutiu-se a importância da gestão de conhecimento como ferramenta que estimula o processo de identificação de oportunidades estratégicas, na ideação de novos projetos com características inovadoras, bem como sua execução.

Concluiu-se que a aplicação do modelo SECl em projetos do segmento da inovação estimulam o sucesso dos mesmos pela retroalimentação da base de conhecimentos institucional com novas experiências vivenciadas ao longo da execução de projetos empreendidos.

Propõe-se a outros autores complementar essa pesquisa com a análise de como gerir de maneira adequada o conhecimento do ambiente externo à organização e sobre sua influência nos projetos de inovação. $A$ despeito da possibilidade da sequência da pesquisa, acredita-se que o trabalho tenha cumprido com os objetivos preestabelecidos.

\section{REFERÊNCIAS}

ASSOCIAÇÃO BRASILEIRA DE NORMAS TÉCNICAS (ABNT). ABNT ISO 21500. São Paulo: ABTN, 2012.

ALVARENGA N.; RIVADÁVIA C. D. Gestão do conhecimento em organizações: proposta de mapeamento conceitual integrativo. 2005.

BALASHOV, A. P. Fundamentals of management. M.: Textbook, 2008.

CANONGIA, C. et al. Foresight, inteligência competitiva e gestão do conhecimento: instrumentos para a gestão da inovação. Gestão \& Produção, v. 11, n. 2, p. 231-238, 2004.

CAVALCANTI, A. M., OLIVEIRA, M. R. G., GRACAS VIEIRA, M.; CAVALCANTI FILHO, A. O característico de inovação setorial: uma métrica para avaliar potencial crescimento de inovação nas micro e pequenas empresas. In: Encontro Nacional de Engenharia de Produção, 32., Bento Gonçalves, 2012. Anais... ENEGEP, 2012.

CORAZZA, R. I.; FRACALANZA, P. S. Sistemas Abertos em Economia: não linearidade e formalização a partir de contribuições da abordagem evolucionista da mudança tecnológica. In: Encontro Nacional de Economia Política, 9., Uberlândia, 2004. Anais... UFU, 2004. Disponível em: http://www.sep.org.br.

DAVENPORT, T.; PRUSAK, L. Conhecimento Empresarial. Rio de Janeiro: Campus, 1998.

DE BES, F.; KOTLER, P. A Bíblia da inovação. São Paulo: Leya, 2011.

DRUCKER, P. F. Management challenges for the 21st century. Routledge, 2007.

FRANCO, D. H.; RODRIGUES, E. A.; CAZELA MOISES, M. Tecnologias e Ferramentas de Gestão. Campinas: Alínea, 2013. 
FRIESL, M. Knowledge acquisition strategies and company performance in young high technology companies. British Journal of Management, v. 23, n. 3, p. 325-343, 2012. Disponível em: http://onlinelibrary.wiley.com/doi/10.1111/j.1467-8551.2011.00742.x/full.

IBM Global Business Services, Global CEO Study 2006. Disponível em: <http://www.ibm.com/bcs/ceostudy>. Acesso em: 01/05/2015.

KEELING, R.; BRANCO, R. H.F. Gestão de Projetos: Uma Abordagem Global. São Paulo: Saraiva, 2012.

KHODYREVA, E. A. Management of innovative educational projects in modern university. Nauchnometodicheskiy elektronnyy zhurnal Kontsept, v. 5, p. 216-220, 2015. Disponível em: http://ekoncept.ru/2015/15172.htm.

LINS, S. Transferindo conhecimento tácito: uma abordagem construtivista. Editora E-papers, 2003.

LUO, $X$. et al. KEMNAD: A knowledge engineering methodology for negotiating agent development. Computational intelligence, v. 28, n. 1, p. 51-105, 2012.

MENEGHETTI, F. K. O que é um Ensaio-Teórico? Revista de Administração Contemporânea, v. 15, n. 2, p. 320-332, 2011.

MORGAN, G. Imagens da organização. São Paulo: Atlas, 2002.

PRADO, D. Maturidade em Gerenciamento de Projetos. 2a. ed. Nova Lima: Editora Falconi, 2010.

PROJECT MANAGEMENT INSTITUTE (PMI). PMBOK Guide: um guia do conhecimento em gerenciamento de projetos. 6 ed. Saraiva, 2017.

SARKAR, S. Empreendedorismo e inovação. Escolar Editora, 2010.

SIMANTOB, M.; LIPPI, R. Guia valor econômico de inovação nas empresas. Rio de Janeiro: Globo, 2003.

SITA, M. et al. Capital Intelectual. A fórmula do Sucesso - Grandes profissionais mostram como investir no desenvolvimento contínuo deste diferencial de sucesso. São Paulo: Ser Mais, 2013.

STEWART, T. A. Capital Intelectual. 3. ed. Rio de Janeiro: Campus, 1998

TAKEUCHI, H.; NONAKA, I. Gestão do conhecimento. Bookman, 2008.

TERRA, J. C. C. Gestão do conhecimento: o grande desafio empresarial. 2005.

VALLE, A. B. et al. Fundamentos do gerenciamento de projetos. Rio de Janeiro: FGV, 2010.

VARGAS, Ricardo V. Gerenciamento de projetos: estabelecendo diferenciais competitivos. 8 ed. São Paulo: Brasport, 2017. 